\title{
Trombólise Endovenosa em Acidente Vascular Cerebral isquêmico: uma revisão de literatura
}

\author{
Thrombolysis Intravenous in Acute Ischemic Stroke: \\ a literature review
}

\section{Trombólisis Endovenosa en Accidente Vascular Cerebral isquêmico: una revisión de la literatura}

\author{
Paula Szymanski ${ }^{1}$, Ivo Marcos Darella Lorenzin Fernandes Neto ${ }^{1}$, \\ Larissa Gabriel Bitencourt ${ }^{1}$, Carlos Fernando dos Santos Moreira2, 3
}

1.Acadêmico(a) do curso de Medicina da Universidade do Extremo Sul Catarinense, Criciúma-SC, Brasil.

2.Docente do curso de Medicina da Universidade do Extremo Sul Catarinense, Criciúma-SC, Brasil.

3.Neurocirurgião no Hospital São José, Criciúma-SC, Brasil.

\begin{abstract}
Resumo
Introdução. O Acidente Vascular Cerebral é uma doença altamente prevalente na população mundial e gera muita morbimortalidade. O subtipo isquêmico é o mais comum ( $85 \%$ de todos os casos) e, se não tratado de maneira rápida e eficaz, produz sequelas e incapacidades permanentes. A Trombólise Endovenosa é uma das modalidades de tratamento agudo mais eficaz se feita no tempo certo, e aprimora a qualidade de vida dos doentes. Método. Revisão de literatura feita nas bases de dados PubMed, SciELO, UpToDate, Biblioteca Nacional em Saúde e Ministério da Saúde, com artigos em inglês, português e espanhol, dos últimos 19 anos. Resultados. Um total de 29 artigos foram selecionados para compor essa revisão de literatura, e tiveram como critérios de seleção o ano de publicação, duplicação de informações e/ou informações incompletas. Conclusões. A Trombólise Endovenosa é um dos principais tratamentos do Acidente Vascular Cerebral isquêmico agudo, pois restaura as áreas de penumbra. Entretanto, deve ser feita em tempo hábil e, para isso, é essencial o atendimento imediato das pessoas com sinais e sintomas do Acidente Vascular Cerebral isquêmico.
\end{abstract}

Unitermos. AVC; isquemia; cérebro; trombólise; alteplase; tomografia

\begin{abstract}
Background. The stroke is a highly prevalent disease and an important cause of morbidity and mortality over the world. The ischemic subtype is the most common one and it represents $85 \%$ of all cases of stroke. If it is not treated in a fast and effective way, the Acute Ischemic Stroke is responsible for several permanent disabilities. The thrombolytic therapy is one of the most effective acute treatment options and if it is performed in time, it allows a better quality of life for patients. Method. A Literature Review was performed, based in data from PubMed, SciELO, UpToDate, Brazilian National Healthy Library and Brazilian Ministry of Health. The articles used in the present study were written in three different languages: English, Portuguese and Spanish and they were all published over the last nineteen years. Results. An amount of 29 articles were selected to compose this Literature Review. The inclusion criteria were year of publication; duplication of information and/or incomplete information. Conclusions. The thrombolytic therapy is one of the most important treatment available for Acute Ischemic Stroke once it allows to restore the penumbra area. However, this therapy must be done in time, and, for this purpose, it is essential to have immediate medical attention when symptoms and signs of stroke begin.
\end{abstract}

Keywords. Stroke; ischemia; brain; thrombolysis; alteplase; tomography 


\section{Resumen}

Introducción. El ictus es una enfermedad de alta prevalencia en la población mundial y genera mucha morbilidad y mortalidad. El subtipo isquémico es el más frecuente $(85 \%$ de todos los casos) y, si no se trata de forma rápida y eficaz, produce secuelas y discapacidades permanentes. La trombólisis intravenosa es una de las modalidades de tratamiento agudo más eficaces si se realiza en el momento adecuado y mejora la calidad de vida de los pacientes. Método. Revisión de la literatura realizada en las bases de datos PubMed, SciELO, UpToDate, Biblioteca Nacional de Salud y Ministerio de Salud, con artículos en inglés, portugués y español, de los últimos 19 años. Resultados. Se seleccionaron un total de 29 artículos para componer esta revisión de literatura, y los criterios de selección fueron el año de publicación, duplicación de información y / o información incompleta. Conclusiones. la trombólisis intravenosa es uno de los principales tratamientos del ictus isquémico agudo, ya que restaura las zonas de penumbra. Sin embargo, debe realizarse de manera oportuna y, para ello, es fundamental la atención inmediata de las personas con signos y síntomas de ictus isquémico.

Palabras clave. Ictus; isquemia; cérebro; trombolisis; alteplasa; tomografía

Trabalho realizado na Universidade do Extremo Sul Catarinense, Criciúma-SC, Brasil.

\section{INTRODUÇÃO}

As doenças cerebrovasculares são a segunda principal causa de óbitos no mundo e, a principal causa de incapacidades ${ }^{1,2}$. A Organização Mundial da Saúde (OMS) afirma que 15 milhões de pessoas/ano são acometidas por essas patologias, sendo que boa parte dos sobreviventes evolui com sequelas físicas e cognitivas ${ }^{3}$.

Atualmente, o Acidente Vascular Cerebral (AVC) é definido como uma injúria cerebral responsável por gerar uma súbita alteração neurológica de características focais e/ou difusas, com duração maior do que 24 horas $^{2}$. Essa condição pode ser determinada por dois diferentes mecanismos: isquêmico - observado em $85 \%$ dos casos ou hemorrágico ${ }^{1,4}$.

O AVC isquêmico (AVCi) advém de uma oclusão, parcial ou total, de um vaso sanguíneo cerebral por uma placa 
aterosclerótica ou por um coágulo vindo do corpo pela circulação ${ }^{5,6}$. A clínica típica do AVCi é a manifestação súbita de déficit neurológico7.

O tratamento do AVCi agudo pode ser feito com Trombólise Endovenosa (TE) com Alteplase, desde que respeitado o período de janela terapêutica do medicamento ${ }^{3,4}$. Tal terapia tem como objetivo reduzir as sequelas e incapacidades residuais do AVCi e, dessa forma, aumentar a qualidade de vida da pessoa acometida ${ }^{1,8}$.

A presente revisão de literatura tem por objetivo compilar diversos conhecimentos científicos de qualidade e atuais sobre o $\mathrm{AVCi}$, desde seu conceito geral, fatores de risco, fisiopatologia, quadro clínico, formas de se fazer o diagnóstico, manejo e tratamento agudo, bem como o prognóstico.

\section{MÉTODO}

Trata-se de uma revisão de literatura realizada nas bases de dados do PubMed, SciELO, UpToDate, Biblioteca Nacional em Saúde e Ministério da Saúde em abril a julho de 2020. Foram utilizados os seguintes descritores: "Stroke", "Cerebral Infarction", "Intravenous Thrombolysis", "Alteplase", "Rehabilitation", "Brain Sequelae"; a busca também foi feita com as palavras-chaves em língua portuguesa e espanhola.

Como critério de inclusão: artigos completos e publicações nas bases de dados oficiais supracitadas, publicadas entre 2001 e 2019. Foram aceitos artigos 
originais, descritivos, revisão de literatura, revisão sistemática e protocolos assistenciais em inglês, português e espanhol. Ao todo, foram utilizados 29 artigos para a confecção desse manuscrito.

\section{RESULTADOS}

Inicialmente foram identificados 45 artigos através da busca, nas bases de dados supracitadas, pelos descritores em inglês, português e espanhol; 13 foram excluídos durante triagem inicial devido a informações incompletas, repetição de dados, e ano muito antigo de publicação. Dos 32 artigos restantes, três não foram incluídos para compor esse manuscrito pois novamente repetiam informações e as regras editoriais limitavam o número de referências. Ao todo, essa revisão de literatura teve como base 29 artigos para a sua escrita (Figura 1).

Figura 1. Fluxograma de seleção dos artigos.
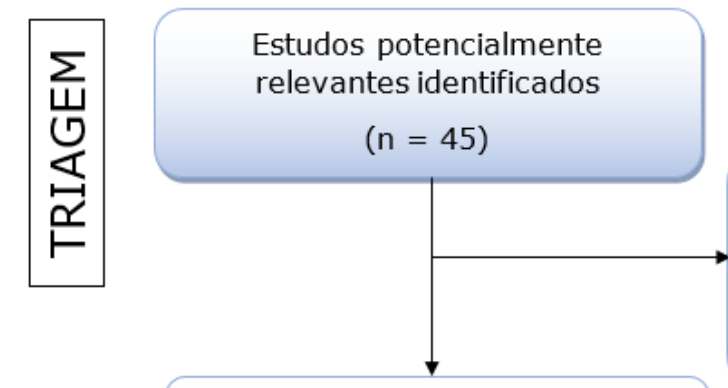

Estudos excluídos pelas razões: $(n=13)$

Repetição de informações; informações incompletas; ano de publicação

Estudos completos para avaliação mais detalhada $(n=32)$

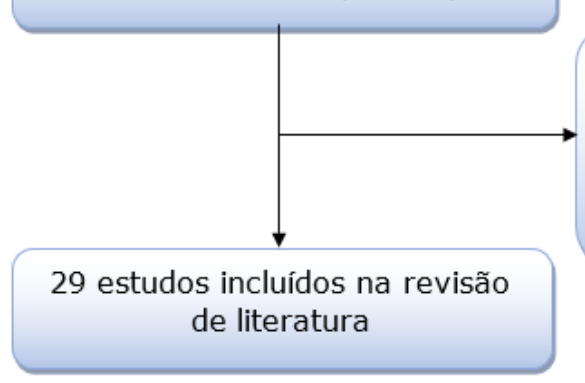

Estudos excluídos pelas razões:

$$
(n=3)
$$

Duplicação de dados e regras para a publicação desse manuscrito 


\section{DISCUSSÃO}

\section{Conceitos}

As doenças cerebrovasculares são consideradas uma das principais causas de morbimortalidade que levam à incapacidade física na população ${ }^{1,2}$. São apontadas como a segunda principal causa de óbitos no mundo e, conforme a Organização Mundial da Saúde (OMS), 15 milhões de pessoas/ano são acometidas por essa patologia, sendo que boa parte dos sobreviventes evolui com sequelas físicas e cognitivas ${ }^{3}$.

O grande representante é o Acidente Vascular Cerebral (AVC), injúria cerebral responsável por gerar uma súbita alteração neurológica de características focais e/ou difusas ${ }^{2}$. Essa condição pode ser determinada por dois diferentes mecanismos: isquêmico - observado em $85 \%$ dos casos ou hemorrágico ${ }^{1,4,9}$.

\section{Fatores de risco}

Dentre os fatores de risco relatados, os principais são: idade maior que 60 anos, hipertensão arterial sistêmica não controlada, história familiar positiva, hiperlipidemia, diabetes mellitus tipo I e II, tabagismo e obesidade ${ }^{9-11}$. Nos indivíduos jovens, há o predomínio de fatores específicos, como: migrâneas com ou sem aura, malignidades, uso de cocaína, gravidez e puerpério, e desordens genéticas/autoimunes ${ }^{11,12}$. Estima-se também que condições ambientais podem ser determinantes para a ocorrência do acidente vascular, como a exposição a poluentes tóxicos ${ }^{12}$. 


\section{Etiologia e fisiopatologia}

O AVC isquêmico (AVCi) advém de uma oclusão, parcial ou total, de um vaso sanguíneo cerebral por uma placa aterosclerótica ou por um coágulo vindo do corpo pela circulação ${ }^{5,6}$. A aterosclerose forma placas gordurosas que levam a estenose do vaso, posterior ulceração das lesões ateroscleróticas e trombose ${ }^{10,11}$. A trombose cerebral é causada pela formação de coágulos internos nas artérias cerebrais e seus ramos ${ }^{10}$.

A área que não recebe sangue resulta em morte neuronal por necrose isquêmica, processo que ocorre entre 4 a 10 minutos; contudo, nas áreas laterais em que o sangue segue através da circulação promovida pelos vasos colaterais, a lesão será menos intensa ${ }^{13}$. Essa área é denominada "zona de penumbra isquêmica", pelo fato de que está no limite entre a morte celular e a retomada das funções ${ }^{14}$. Caso instaurado o tratamento precoce, haverá uma alta taxa de reversão isquêmica ${ }^{2,13,15}$.

\section{Apresentação clínica}

A clínica típica do AVCi é a manifestação súbita de déficit neurológico ${ }^{7}$. O principal sintoma é a hemiplegia, caracterizada pela perda do controle motor voluntário do hemicorpo contralateral à lesão encefálica, que se instala em

minutos ou horas e leva a alterações no controle postural e no equilíbrio 2,5,16,17. A localização da lesão na topografia cerebral e a extensão do dano, irão determinar o quadro neurológico de cada paciente com $\mathrm{AVCi}^{5,16}$. A sintomatologia 
piora na coexistência de isquemia e sangramento intracerebral ${ }^{1}$. A oclusão de diferentes artérias origina diferentes síndromes vasculares, cada qual com sinais e sintomas clínicos característicos como: diminuição de força e/ou sensibilidade contralateral, afasia ou disartria, alteração de consciência, confusão e perda de memória momentânea ${ }^{10,16}$.

Os sinais e sintomas clínicos advindos do AVCi geram prejuízos ao limitar a realização das atividades de vida diária, contribuem para a restrição e o isolamento social por parte do doente e, consequentemente a piora da qualidade de vida ${ }^{18}$.

\section{Diagnóstico}

O diagnóstico do AVC é clínico-radiológico, composto por história e exame físico que detém até $92 \%$ de sensibilidade associado a um exame de imagem para diferenciar o AVCi do AVC hemorrágico (AVCh) 1,13,14.

Para quantificar a sintomatologia do paciente utiliza-se a "National Institutes of Health Stroke Scale" (NIHSS), escala de avaliação intra hospitalar, composta por 15 itens, sendo usada para determinar a gravidade do quadro clínico e para predizer prognósticos ${ }^{14}$.

A Tomografia Computadorizada (TC) sem contraste é o método de escolha ideal para a avaliação inicial do AVCi pois demonstra sinais precoces de isquemia nas primeiras três horas do início dos sintomas e demanda menor tempo/custo para realização ${ }^{1,13,19}$. Ela apresenta a lesão isquêmica como 
uma hipodensidade do parênquima cerebral, devido ao edema isquêmico, que não se impregna pelo contraste, ressalta o apagamento localizado dos sulcos e cisternas e, também pode mostrar o sinal da artéria cerebral média hiperdensa, sendo um achado precoce de infarto cerebral ${ }^{19-}$ 21.

A Ressonância Magnética (RM) possui sensibilidade maior que a TC para a detecção de AVCi agudo, todavia é mais cara, demanda mais tempo, tem baixa disponibilidade intra-hospitalar e não pode ser feita em pessoas que possuem artefatos metálicos no corpo ${ }^{14}$.

Outros exames laboratoriais e de imagem com maior acurácia podem ser feitos para investigar a etiologia do AVCi e para eliminar diagnósticos diferenciais como convulsões, hipoglicemia, tumores e infeções sistêmicas; entretanto, eles costumam serem realizados durante a internação do paciente e não na sala de emergência3,8,14,19.

\section{Manejo}

A avaliação inicial deve priorizar: tempo comprovado do início dos sintomas, sinais vitais e exame neurológico com escala de coma de Glasgow e de NIHSS ${ }^{4}$. A TC e a avaliação dos critérios de inclusão e exclusão para o tratamento com a terapia trombolítica devem ser prontamente realizadas ${ }^{3}$.

O National Intitute of Neurological Disorders and Stroke traz recomendações mundiais para os candidatos potenciais ao uso de trombólise endovenosa (TE), com relação aos tempos ideais: 10 minutos desde a admissão até a avaliação 
médica; 25 minutos da admissão até a realização da TC craniana; 45 minutos da admissão até a interpretação da TC (tempo porta-tomografia); 60 minutos da admissão até a infusão do Alteplase (rt-PA) (tempo porta-agulha); 15 minutos para a disponibilidade do neurologista; 2 horas para a disponibilidade do neurocirurgião; 3 horas da admissão até a monitoração no leito ${ }^{4}$. Atualmente, são aceitos para tratamento com $\mathrm{TE}$, casos de $\mathrm{AVCi}$ de até 4,5 horas de evolução22.

É de extrema importância mencionar que, além do $A \vee C i$, outras comorbidades como a hipertensão, coexistem no mesmo paciente e, por isso, é dever do médico manejá$\operatorname{las}^{4,8}$.

\section{Tratamento}

O procedimento de TE é feito com o fim de dissolver um trombo ou um êmbolo na corrente sanguínea 5 . O objetivo imediato da terapia trombolítica é restaurar o fluxo sanguíneo nas regiões isquêmicas, porém ainda não infartadas $^{22}$. Ele atua convertendo o plasminogênio em plasmina, que solubiliza a fibrina e degrada o fibrinogênio e os fatores V, VIII, IX, XI e XII ${ }^{23}$. A curto e longo prazo traz benefícios ao paciente, reduz suas incapacidades, sequelas e taxa de mortalidade ${ }^{22}$.

A administração do rt-PA deve ser discutida com os familiares e/ou responsáveis, esclarecido os riscos e benefícios do tratamento e registrada a decisão por escrito no prontuário do paciente ${ }^{4,8}$. A TE não é recomendada 
quando o início do AVCi não é preciso, como no caso de pessoas que o tiveram dormindo, pois, pode ser que a janela terapêutica não seja a mesma do medicamento logo, não haverá benefícios ${ }^{19}$.

O Alteplase possui uma série de critérios que visam incluir ou excluir o paciente na terapia trombolítica. Segundo Manual de Rotinas para Atenção ao $\mathrm{AVC}^{3}$, são eles:

"Critérios de inclusão: AVC isquêmico em qualquer território encefálico; possibilidade de se iniciar a infusão do rt-PA dentro de 4,5 horas do início dos sintomas (o horário do início dos sintomas deve ser corretamente estabelecido, pois caso os sintomas forem observados ao acordar, devese considerar o último horário no qual o paciente foi observado normal); TC do crânio ou RM sem evidência de hemorragia; idade superior a 18 anos.

Critérios de exclusão: Uso de anticoagulantes orais com tempo de protrombina (TP) com índice internacional normalizado (RNI) >1,7; uso de heparina nas últimas 48 horas com tempo de tromboplastina parcial ativada (TTPA) elevado; AVC isquêmico ou traumatismo cranioencefálico grave nos últimos três meses; história pregressa de hemorragia intracraniana ou de malformação vascular cerebral; TC de crânio com hipodensidade precoce $>1 / 3$ do território da artéria cerebral média ( $A C M)$; pressão arterial (PA) sistólica $\geq 185 \mathrm{mmHg}$ ou PA diastólica $\geq 110 \mathrm{mmHg}$ (em três ocasiões, com 10 minutos de intervalo) refratária ao tratamento anti-hipertensivo; melhoria rápida e completa dos sinais e sintomas no período anterior ao início da 
trombólise; deficits neurológicos leves (sem repercussão funcional significativa); cirurgia de grande porte ou procedimento invasivo nos últimos 14 dias; punção lombar nos últimos 7 dias; hemorragia geniturinária ou gastrointestinal nos últimos 21 dias ou história de varizes esofagianas; punção arterial em local não compressível na última semana; coagulopatia com TP prolongado ( $R N I>1,7)$, TTPA elevado ou plaquetas $<100.000 / \mathrm{mm} 3$; glicemia $<50 \mathrm{mg} /$ dl com reversão dos sintomas após a correção; evidência de endocardite ou êmbolo séptico, gravidez; Infarto do miocárdio recente (três meses) - contraindicação relativa; suspeita clínica de hemorragia subaracnoide ou dissecção aguda de aorta".

Posterior a análise dos critérios de inclusão e exclusão, devem ser feitos dois acessos venosos periféricos e administrar o rt-PA na dose de $0,9 \mathrm{mg} / \mathrm{kg}$ com a dose máxima de $90 \mathrm{mg}$, sendo que $10 \%$ dessa dose deve ser feita no primeiro minuto e, o restante em 60 minutos, de preferência com bomba de infusão ${ }^{3}$. A diluição do rt-PA é de $1 \mathrm{mg} / \mathrm{ml}^{3,4}$.

Durante a infusão do rt-PA e nas 24 horas posteriores, o paciente deve ser mantido em uma unidade de AVC ou em uma Unidade de Terapia Intensiva (UTI), para a detecção de qualquer mudança do estado neurológico, aferição de sinais vitais, observação comportamental e, com disponibilidade multiprofissional e de exames de imagem ${ }^{4}$.

Os benefícios esperados com o uso da TE são: redução do tempo de recuperação da capacidade de deambular; redução do número de pacientes com complicações e mortes 
pelo $\mathrm{AVCi}$; e redução do grau de incapacidade um ano após o tratamento ${ }^{1,8}$.

Recentemente, a terapia de reperfusão cerebral com a Trombectomia Mecânica (TM) vem ganhando espaço no tratamento do $\mathrm{AVCi}^{24}$. Os pacientes com $\mathrm{AVCi}$ elegíveis ao tratamento com rt-PA, possuem uma janela terapêutica de até 4,5 horas; já com a TM, esse intervalo se estendeu, e o paciente pode ser tratado com essa terapia se for abordado dentro de 6 a 24 horas após a última vez que foi visto consciente, independentemente de ter feito TE antes para o mesmo evento isquêmico ${ }^{25}$. É válido prezar, assim como a $T E$, pelo tratamento precoce ${ }^{24}$.

A TM é mais precisamente indicada aos AVCi com oclusão de artéria na circulação anterior e pode ser feita isoladamente ou em pacientes que já foram submetidos a TE anterior no mesmo $A V C i$, a qual não foi resolutiva ou é contraindicada ${ }^{26}$. A TM arterial é superior ao tratamento padrão com $\mathrm{TE}$, porém ainda não está disponível em todos os centros de saúde assim como, os profissionais habilitados para executar esse procedimento são $\operatorname{raros}^{27}$.

\section{Prognóstico}

O prognóstico do paciente tem total relação com o tempo: quanto mais rápido a pessoa com AVCi for atendida, mais rápido será feita a $T E$, menor a área de infarto, mais área de penumbra é salva e, menos sequelas se desenvolverão, por isso: "time is brain"28. 
As sequelas e as incapacidades físicas, motoras ou cognitivas, interferem na qualidade de vida do paciente e da família ${ }^{11,29}$. Atualmente, até $90 \%$ dos sobreviventes de um AVC desenvolvem alguma incapacidade, e 60\% desses permanecem com disfunção motora permanente ${ }^{17,18}$. Por isso, a reabilitação de sucesso é promovida pelo trabalho de uma equipe multidisciplinar com foco no paciente e nas orientações dadas às pessoas com as quais ele convive 2,5,11,17.

Devido à dificuldade no acesso ao tratamento e à possibilidade de a doença cursar com sequelas, torna-se vital atuar na prevenção ${ }^{2}$. Isso consiste na associação de medidas farmacológicas e não farmacológicas que permitam controlar os principais fatores de risco desencadeantes dessa cerebrovasculopatia 2,5,12,29. Por isso, ressalta-se novamente a importância das ações preventivas, pois sozinhas podem reduzir em até $80 \%$ o risco de desenvolver AVC isquêmico ou a recorrência do mesmo ${ }^{11}$.

\section{CONCLUSÃO}

O AVCi é uma cerebrovasculopatia de alta morbimortalidade e, está associado a diversos fatores de risco comuns na população mundial. As consequências dessa injúria vascular encefálica são inúmeras, variadas, e se não tratadas adequadamente, de maneira rápida, tornam-se irreversíveis. A TE é muito eficaz no tratamento do AVCi agudo, desde que feita no tempo hábil da janela terapêutica. É prezado pela rapidez na assistência médica, visando 
sempre reduzir sequelas e aumentar a qualidade de vida dos acometidos. De tal forma, é importante a conscientização populacional sobre os principais sintomas de um AVCi, bem como chamar por ajuda sempre que identificá-los, e assim, o paciente conseguirá receber um tratamento eficiente e resolutivo.

\section{REFERÊNCIAS}

1.Brasil. Ministério da Saúde (endereço na internet). Portaria GM/MS no 664, de 12 de abril de 2012. Protocolo clínico e diretrizes terapêuticas: trombólise no acidente vascular cerebral isquêmico agudo. Brasília: Ministério da Saúde; 2012 (acessado em 2020). Disponível em:

https://bvsms.saude.gov.br/bvs/saudelegis/gm/2012/PRT0664 120 4 2012.html

2.Sá BP, Grave MT, Périco E. Perfil de pacientes internados por acidente vascular cerebral em hospital do Vale do Taquari/RS. Rev Neurocienc 2014;22:381-7. https://doi.org/10.34024/rnc.2014.v22.8077

3.Brasil. Ministério da Saúde. Secretaria de Atenção à Saúde. Manual de rotinas para atenção ao AVC. Brasília: Ministério da Saúde; 2013, $54 \mathrm{p}$.

https://bvsms.saude.gov.br/bvs/publicacoes/manual rotinas para at encao avc.pdf

4.Raffin CN, Gagliardi RJ, Massaro AR, Fernandes JG, Bacellar AL, Fábio $\mathrm{SR}$, et al. Primeiro consenso brasileiro para trombólise no acidente vascular cerebral isquêmico agudo. Arq Neuropsiquiatr 2002;60:67580. https://doi.org/10.1590/S0004-282X2002000400032

5.Cancela DM. O acidente vascular cerebral: classificação, principais consequências e reabilitação (dissertação). Portugal: Universidade Lusíada do Porto; 2008.

https://www.psicologia.pt/artigos/textos/TL0095.pdf

6. Freitas GR. Tratamento agudo do acidente vascular cerebral isquêmico: trombolíticos e antitrombolíticos. Rev SOCERJ 2001;14:7481.

http://sociedades.cardiol.br/socerj/revista/2001_01/a2001_v14_n01 art11.pdf

7.Moulin S, Leys D. Stroke mimics and chameleons. Curr Opin Neurol 2019;32:54-9. https://doi.org/10.1097/WC0.0000000000000620

8.Silva GS, Gomes DL, Massaro AR. Tratamento da fase aguda do acidente vascular cerebral isquêmico. Rev Neurocienc 2005;13:39-49. https://doi.org/10.34024/rnc.2005.v13.8844

9.Guzik A, Bushnell C. Stroke epidemiology and risk factor management. Continuum (Minneap Minn) 2017;23:15-39. https://doi.org/10.1212/CON.0000000000000416 
10.Araujo LP, Souza GS, Dias PL, Nepomuceno RM. Principais fatores de risco para o acidente vascular encefálico e suas consequências: uma revisão de literatura. REINPEC 2017;1:284-96.

https://doi.org/10.20951/2446-6778/v3n1a20

11.Ekker MS, Bool EM, Singhal AB, Tan KS, Debette S, Tuladhar AM, et al. Epidemiology, aetiology, and management of ischaemic stroke in young adults. Lancet Neurol 2018;17:790-801.

https://doi.org/10.1016/S1474-4422(18)30233-3

12.Towfighi A, Hill VA. Modifiable risk factors for stroke and strategies for stroke prevention. Semin Neurol 2017;37:237-58. https://doi.org/10.1055/s-0037-1603685

13.Giannini MA, Yugar-Toledo JC, Vilela-Martin JF. Emergência hipertensiva e acidente vascular cerebral isquêmico e hemorrágico: conceitos atuais de tratamento. Rev Bras Hipertensão 2014;21:17783. $\quad$ https://docs.bvsalud.org/biblioref/2018/03/881314/rbh-

v21n4 177-

183.pdf\# : : text=ACIDENTE\%20VASCULAR $\% 20$ ENCEF\%C3\%81LICO \%20NA\%20CRISE,em\%20AVE\%20hemorr\%C3\%A1gico\%20e\%20isq u\%C3\%AAmico

14.Yew KS, Cheng EM. Acute stroke diagnosis. Am Fam Phys 2009;80:33-40. https://pubmed.ncbi.nlm.nih.gov/19621844/

15.Lorente L, Martín MM, Abreu-González P, Sabatel R, Ramos L, Argueso $\mathrm{M}$, et al. Non-survivor patients with malignant middle cerebral artery infarction showed persistently high serum malondialdehyde levels. BMC Neurology 2019;19:1-6. https://doi.org/10.1186/s12883$\underline{019-1479-z}$

16. Melo LS, Emerick LM, Alves PN, Rocha TB, Goveia VR, Guimarães $\mathrm{GL}$, et al. Acidente vascular cerebral: achados clínicos e principais complicações. RAS 2016;14:45-53.

https://doi.org/10.13037/ras.vol14n48.3685

17.Piassaroli CAP, Almeida GC, Luvizotto JC, Suzan AB. Modelos de reabilitação fisioterápica em pacientes adultos com sequelas de AVC isquêmico. Rev Neurocienc 2011;20:128-37.

https://doi.org/10.34024/rnc.2012.v20.10341

18.Scalzo PL, Souza ES, Moreira AGO, Vieira DAF. Qualidade de vida em pacientes com acidente vascular cerebral: clínica de fisioterapia Puc Minas Betim. Rev Neurocienc 2010;18:139-44.

https://doi.org/10.34024/rnc.2010.v18.8480

19.European Stroke Initiative Executive Committee, EUSI Writing Committee, Olsen TS, Langhorne P, Diener HC, Hennerici M, et al. European Stroke Initiative Recommendations for Stroke Management - update 2003. Cerebrovasc Dis 2003;16:311-37.

https://doi.org/10.1159/000072554

20.Abreu TT. Sinais tomográficos precoces do AVC isquémico. Rev Med Int 2002;9:45-51. https://www.spmi.pt/revista/vol09/sinaisavc.pdf 21.Sacco RL, Kasner SE, Broderick JP, Caplan LR, Connors J], Culebras $A$, et al. An updated definition of stroke for the 21st century: a statement for healthcare professionals from the American Heart 
Association/American Stroke Association. Stroke 2013;44:2064-89. https://doi.org/10.1161/STR.0b013e318296aeca

22.Powers WJ, Rabinstein AA, Ackerson T, Adeoye OM, Bambakidis NC, Becker K, et al. Guidelines for the early management of acute ischemic stroke: 2019 update to the 2018 Guidelines for healthcare professionals from the American Heart Association/American Stroke Association. Stroke 2019;50:e344-418.

https://doi.org/10.1161/STR.0000000000000211

23. Henderson SJ, Weitz JI, Kim PY. Fibrinolysis: strategies to enhance the treatment of acute ischemic stroke. J Thromb Haemost 2018;16:1932-40. https://doi.org/10.1111/ith.14215

24.Campbell BCV, Donnan GA, Less KR, Hacke W, Khatri P, Hill MD, et al. Endovascular stent thrombectomy: the new standard of care for large vessel ischaemic stroke. Lancet Neurol 2015;14:846-54. https://doi.org/10.1016/S1474-4422(15)00140-4

25.Jadhav AP, Desai SM, Kenmuir CL, Rocha M, Starr MT, Molyneaux $\mathrm{BJ}$, et al. Eligibility for endovascular trial enrollment in the 6- to 24hour time window: analysis of a single comprehensive stroke center. Stroke 2018;49:1015-7.

https://doi.org/10.1161/STROKEAHA.117.020273

26. Nogueira RG, Jadhav AP, Haussen DC, Bonafe A, Budzik RF, Bhuva $\mathrm{P}$, et al. Thrombectomy 6 to 24 hours after stroke with a mismatch between deficit and infarct. New Eng J Med 2018;378:11-21. https://doi.org/10.1056/NEJMoa1706442

27.Berkhemer OA, Fransen PSS, Beumer D, van den Berg LA, Lingsma $\mathrm{HF}$, Yoo AJ, et al. A randomized trial of intraarterial treatment for acute ischemic stroke. New Eng J Med 2015;372:11-20. https://doi.org/10.1056/NEJMoa1411587

28. Michaels AD, Spinler AS, Leeper B, Ohman EM, Alexander KP, Newby LK, et al. Medication errors in acute cardiovascular and stroke patients: a scientific statement from the American Heart Association. Circulation 2010;121:1664-82. https://doi.org/10.1161/CIR.0b013e3181d4b43e

29. Henriques M, Henriques J, Jacinto J. Acidente vascular cerebral no adulto jovem: a realidade num centro de reabilitação. Rev SPMFR 2015;27:9-13. http://dx.doi.org/10.25759/spmfr. 180 